\title{
Comparison of Subjective and Objective Sleep Estimations in Patients with Bipolar Disorder and Healthy Control Subjects
}

\author{
Philipp S. Ritter, Cathrin Sauer, Steffi Pfeiffer, Michael Bauer, and Andrea Pfennig \\ Klinik und Poliklinik für Psychiatrie und Psychotherapie, Universitätsklinikum Carl Gustav Carus an der Technischen \\ Universität Dresden, Fetscherstraße 74, 01307 Dresden, Germany
}

Correspondence should be addressed to Philipp S. Ritter; philipp.ritter@uniklinikum-dresden.de

Received 1 April 2016; Revised 24 August 2016; Accepted 29 September 2016

Academic Editor: Chih Y. Huang

Copyright (c) 2016 Philipp S. Ritter et al. This is an open access article distributed under the Creative Commons Attribution License, which permits unrestricted use, distribution, and reproduction in any medium, provided the original work is properly cited.

Background. Several studies have described but not formally tested discrepancies between subjective and objective measures of sleep. Study Objectives. To test the hypothesis that patients with bipolar disorder display a systematic bias to underestimate sleep duration and overestimate sleep latency. Methods. Actimetry was used to assess sleep latency and duration in 49 euthymic participants (bipolar $=21$; healthy controls $=28$ ) for 5-7 days. Participants simultaneously recorded estimated sleep duration and sleep latency on a daily basis via an online sleep diary. Group differences in the discrepancy between subjective and objective parameters were calculated using $t$-tests and corrected for multiple comparisons. Results. Patients with bipolar disorder significantly underestimated their sleep duration but did not overestimate their sleep latency compared to healthy controls. Conclusions. Studies utilizing diaries or questionnaires alone in patients with bipolar disorders may systematically underestimate sleep duration compared to healthy controls. The additional use of objective assessment methods such as actimetry is advisable.

\section{Introduction}

Bipolar disorders are severe mental disorders affecting approximately $1-3 \%$ of the population [1]. There is cumulating evidence that bipolar disorder and disturbances of sleep regulation are deeply intertwined on a phenomenological and neurobiological level [2]. Epidemiological research has shown symptoms of insomnia to be a risk factor for the subsequent development of bipolar disorder [3] and various studies have demonstrated patients to suffer from varying degrees of insomnia even when euthymic $[4,5]$.

Most research regarding sleep in bipolar disorders is conducted using questionnaires or sleep diaries alone [6]. However, there is evidence that patients suffering from bipolar disorder may have deficits in precise time estimation [7] and indeed those studies utilizing objective measures of sleep duration and sleep latency such as actimetry or polysomnography have produced discrepancies between objective and subjective markers with patients often underestimating their sleep duration and overestimating sleep latency $[2,8,9]$. The literature suggests that this discrepancy is most prominent with regard to sleep onset latency and sleep duration.
However, while this issue has been exhaustively investigated in patients with primary insomnia [10], there are-to the best of our knowledge-no studies specifically analysing the mismatch of these two parameters in terms of absolute time in bipolar disorder. Assessing the absolute mismatch is important, because the pure correlation of objective and subjective data will reveal a perfect correlation even if there is a systematic mismatch between the two.

We therefore hypothesised that patients suffering from bipolar disorder have a general tendency to underestimate sleep duration and overestimate sleep latency and conducted a study comparing sleep parameters generated by actigraphy with simultaneously completed sleep diaries. The sample utilized was part of a previously published study on early recognition of bipolar disorder [11].

\section{Methods}

We recruited 22 euthymic outpatients from a specialist outpatient clinic for patients with bipolar disorder at the University Hospital Dresden with a diagnosis of bipolar disorder and 28 healthy volunteers via advertisements on campus and other 
public spaces. One bipolar participant was excluded due to missing diary information.

The recruitment period extends from January 2011 until January 2012.

After providing informed consent, all participants were examined by an experienced psychiatrist using the SCID 1 and 2 [12] for DSM IV as well as the Montgomery-Asberg Depression Rating Scale (MADRS) [13, 14] and Young-Mania Rating Scale (YMRS) [15]. Euthymia was liberally defined as MADRS $\leq 15$ and YMRS $\leq 10$ at screening. The cut-off scores were based on empirical evidence correlating these values to the Clinical Global Impression Scale (CGI-S) in a large cohort of symptomatic bipolar patients. The individual values correspond to a CGI impression of "mildly ill" [16].

Control subjects with first-degree relatives with a major mental disorder except for dementia were excluded. Participants were also excluded if they had a known diagnosed sleep disorder such as narcolepsy, restless leg syndrome, or obstructive-sleep-apnea syndrome. Other medical conditions likely to interfere with sleep (i.e., chronic back pain) and disruptions in circadian rhythms caused by shiftwork, recent (past 3 months) transmeridian flights, or other factors likely to interfere with sleep (i.e., newborn child, significant life events) were considered reasons for exclusion. The presence of symptoms of insomnia was no reason for exclusion.

Participants were required to abstain from the use of illicit drugs for the duration of the study. Sedating medication (i.e., sedating antipsychotics) was permitted if taken regularly for at least one month (Table 2). Patients receiving Benzodiazepines were excluded. The amount of coffee (cups) and alcohol (units) intake was recorded on a daily basis.

The SomnoWatch plus@ is microelectromechanical-system (MEMS) based and contains a light sensor and an event marker. All participants wore an actimeter (SomnoWatch plus@), Somnomedics, Germany) for 5 to 7 days and simultaneously completed an online sleep diary (https://www.limesurvey.org/) including questions on estimated sleep duration and estimated sleep latency (Supplement 1 in Supplementary Material available online at http://dx.doi.org/10.1155/2016/ 4031535). All measurements included one weekend. Participants were required to wear the actimeter at all times except when in contact with water. In the evening the time at which participants lay down to sleep ("lights off") probands were instructed to press an event marker on the actimeter. The same was required in the morning when participants awoke and wanted to stay awake ("lights on"). The actimeter and algorithm utilized have been validated and show reasonable validity compared to polysomnography [17]. Time in Bed (TIB) is defined as the time between the two event markers. Sleep duration is defined as TIB with sleep latency and wake periods subtracted. The sleep parameters were calculated using DominoLight $\odot$ software (Somnomedics, Germany). All data was analysed using SPSS $\odot$ Vs.21.

The self-rating instruments Beck Depression Inventory (BDI) [18] and Altman Self-Rating Scale for Mania (ASRM) [19] were completed as part of the online diary on the first day of assessment.

The differences between estimated sleep parameters and actimetrically determined sleep parameters were calculated on a daily basis for every individual participant $\left(\Delta_{\text {duration }}\right.$; $\Delta_{\text {latency }}$ ). Group mean and SD were calculated from individual data across the 5-7 days. Group differences were analysed using $t$-test for independent means. The significance level was set at 0.05 and Bonferroni correction for multiple testing was applied.

Normal distribution was assessed using the KolmogorovSmirnov test. Differences between groups in coffee and alcohol consumption were calculated using $t$-tests and Pearson correlation to assess association with sleep parameters of interest.

As a possible confounder correlations between age and all related subjective and objective sleep parameters were calculated. In addition, Spearman correlations with ASRM and BDI with subjective and objective sleep parameters were calculated.

The study was approved by the ethics committee of the medical faculty of the University of Dresden (Medizinische Fakultät der TU Dresden).

\section{Results}

Patients with bipolar disorder were approximately four years older on average, though this difference was not significant $(p=0.1)$ (Table 1). The majority of patients had bipolar I disorder (Table 2).

Patients with bipolar disorder generally underestimated their sleep duration by 55.05 minutes while healthy control subjects underestimated sleep duration by 4.38 minutes (Figure 1). The between-group difference was significant (corrected $p=0.02$ ). Patients with bipolar disorder overestimated their sleep latency by $3.02 \mathrm{~min}$ while healthy controls overestimated their sleep latency by $1.12 \mathrm{~min}$ (Figure 2). The group differences were not statistically significant (corrected $p=0.32$ ).

Approximately a third of participants consumed moderate amounts of alcohol prior to bedtime. The amount of alcohol consumed was slightly higher in the control group ( control $=2.93$ units $/$ day; bipolar $=2.03$ units/day; $p=0.40$ ). The amount of coffee consumed was slightly elevated in the bipolar group (control 1.41 cups/day; bipolar 2.01 cups/day; $p=0.08)$. There were no significant correlations between alcohol or coffee consumption and any of the actigraphy or sleep diary parameters of interest.

The BDI score was marginally but significantly elevated in the bipolar group. However, there were no correlations between age, BDI, or ASRM and any of the actigraphy or sleep diary parameters of interest.

\section{Discussion}

The results confirm the observation that patients with bipolar disorder have a systematic tendency to underestimate their sleep duration. They do not appear to misjudge their sleep latency to a significant degree. Previous comparisons of objective and subjective sleep parameters have largely relied on correlations $[8,20]$. However while correlating data will give a good measure of covariance it will not detect a systematic tendency to over- or underestimate a certain measure 
TABLE 1: Sociodemographic variables.

\begin{tabular}{lcccc}
\hline Group & & Bipolar subjects $(n=21)$ & Control subjects $(n=28)$ & $\begin{array}{c}\text { Between-group difference } \\
p \text { value }\end{array}$ \\
\hline Age & Mean (SD) & $32.95(10,19)$ & $28.65(7,3)$ & $0,099^{\mathrm{a}}$ \\
Sex & \% female & $13(61,9 \%)$ & $16(57,1 \%)$ & $0,737^{\mathrm{b}}$ \\
Average years in education & Mean (SD) & $13.25(1.53)$ & $13.49(1.87)$ & $0.64^{\mathrm{a}}$ \\
BDI & Mean (SD) & $4.95(5.7)$ & $0.68(1.49)$ & $>\mathbf{0 . 0 1}^{\mathrm{a}}$ \\
ASRM & Mean (SD) & $1.5(1.57)$ & $0.96(1.55)$ & $0.44^{\mathrm{a}}$ \\
\hline
\end{tabular}

BDI: Beck Depression Inventory; ASRM: Altman Self-Rating Scale for Mania.

${ }^{\mathrm{a}}$-test; ${ }^{\mathrm{b}}$ chi-squared test.

TABLE 2: Clinical variables.

\begin{tabular}{lcc}
\hline $\begin{array}{l}\text { Bipolar subjects } \\
\text { Age of onset }\end{array}$ & Mean (SD) & $19,38(7,18)$ \\
$\begin{array}{l}\text { Number of depressive episodes } \\
\text { Number of manic episodes }\end{array}$ & Mean (SD) & $7,05(8,67)$ \\
$\quad$ Mean (SD) & $4,95(7,17)$ \\
\hline Medication $^{*}$ & Total & $19 / 2$ \\
\hline Lithium & Total & $\%$ \\
Valproic acid & 12 & 57,14 \\
Lamotrigine & 9 & 42,86 \\
Quetiapine & 3 & 14,29 \\
Other atypical antipsychotics & 9 & 42,86 \\
(olanzapine, aripiprazole, clozapine) & 4 & 19,05 \\
\hline
\end{tabular}

${ }^{*}$ Most patients received $>1$ substance.

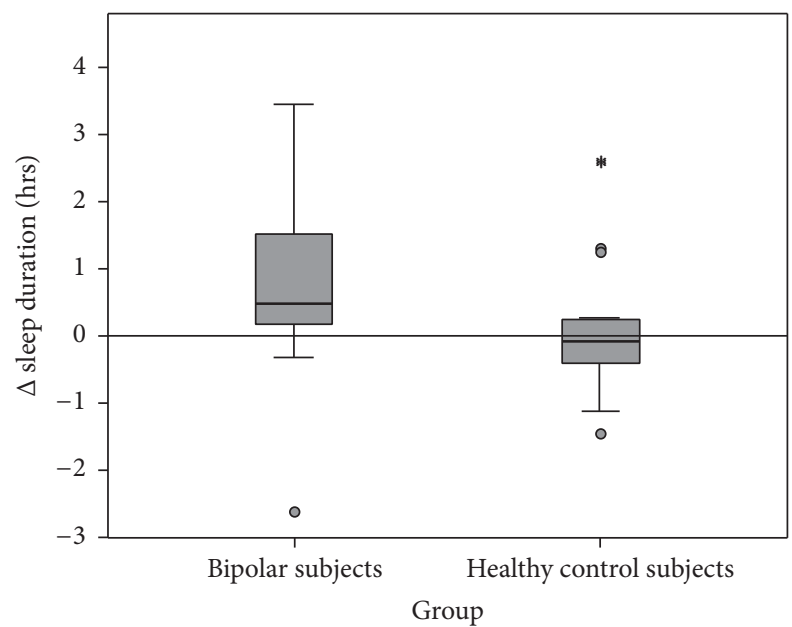

Figure 1: $\Delta$ sleep duration. Discrepancy between actimetrically determined values and sleep diary. Positive values indicate subjective underestimation of sleep duration.

within one group if this over- or underestimation is proportionally constant. To our knowledge, this study is the first to specifically compare absolute values of sleep parameters between patients and controls. A particular strength of this study is the fact that participants were required to estimate their sleep parameters on a daily basis thereby avoiding recall bias.

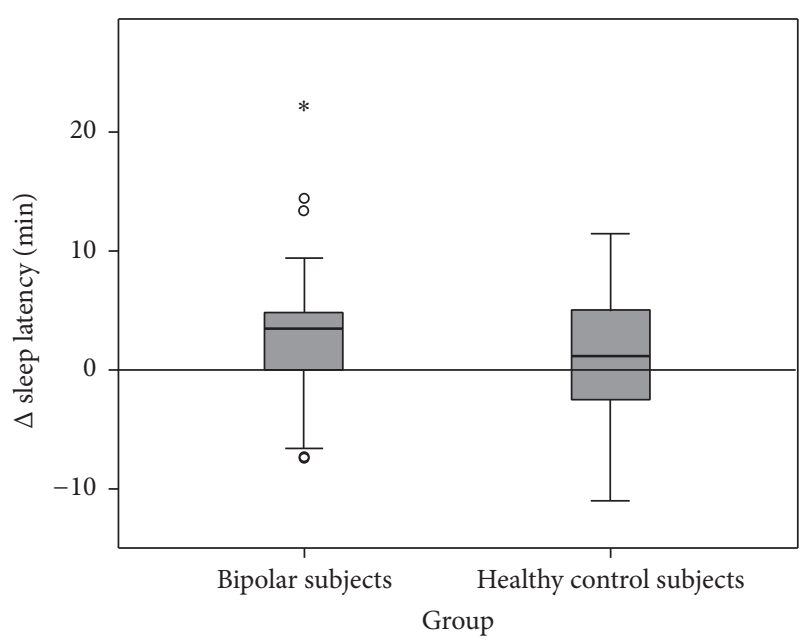

FIGURE 2: $\Delta$ sleep latency. Discrepancy between actimetrically determined values and sleep diary. Positive values indicate subjective overestimation of sleep latency.

If the results are confirmed in other samples, certain implications for research on sleep in bipolar disorder will arise. Although diaries are undoubtedly beneficial, convenient, and uncomplicated in characterizing sleep quality overall, estimating sleep duration from diaries or questionnaires alone ought to be avoided especially when making comparisons to healthy controls, because it has to be assumed that patients with bipolar disorder will systematically underestimate their sleep duration. Currently sleep duration is most frequently assessed by questionnaire alone but the results may be biased. This is of particular importance since a substantial portion of the evidence linking bipolar disorder to insomnia is based on questionnaire data. Other methods for assessing sleep duration such as actigraphy have become more available and convenient to use. Due to improvements in memory and battery capacity, many of the currently available devices can assess circadian rhythms and sleep for 7 days or more without recharging and have significantly reduced in size, thus improving suitability for patients. The results should encourage the use of actigraphy or similar technologies for the assessment of sleep duration in bipolar disorder to improve the reliability of results.

The study has several limitations. Firstly, the actimetry settings were very sensitive to optimize detection of 
sleep latency and wake episodes during sleep. The absolute time of sleep duration may therefore have been slightly underestimated. However there is no reason to believe that this should affect bipolar patients differently from healthy controls. Secondly, the sample size is comparable to other studies utilizing actimetry [21] but larger samples are generally desirable and group differences in $\Delta_{\text {latency }}$ may not have been detected due to lack of power. Thirdly, participants were allowed to consume coffee and alcohol, which may bias judgement. However no significant differences were found in between groups and overly harsh restrictions on permitted behaviours reduce the external validity of the results. Fourthly, bipolar patients had slightly elevated rates of depressive symptoms. These can certainly impair correct judgement and presumably this also includes duration of sleep. However the absolute level was low and no correlations with the sleep parameters of interest could be found suggesting that there was no systematic bias induced by higher rates of depressive symptoms. Again overly harsh restrictions on psychopathology reduce the external validity, since low grade residual symptoms are common in euthymic bipolar disorder [22]. Fifthly, the use of volunteers always involves the risk of recruiting a sample that is not genuinely representative of the population. There is currently no obvious method to avoid or even quantify this potential bias. Sixthly, the reliability of actimetry improves with duration of measurement, and some [23] but not all authors [24], therefore, recommend a longer duration of measurement. Seventhly, all patients received psychotropic medication. This limitation is applicable to all studies studying sleep or chronobiological aspects of bipolar disorder and it is certainly imaginable that judgement of sleep duration may be altered by psychotropic medication. Since it would be ethically unjustified to discontinue medication and patients with bipolar disorder who regularly attend outpatient clinics commonly receive medication, there is no simple solution to this dilemma. The sample size is too small to study the individual effects of the different medications used.

This study adds to the evidence that patients with bipolar disorder systematically underestimate their sleep duration. Since a multitude of studies have been published on the alterations in the sleep of bipolar patients as assessed by questionnaire these results ought to be evaluated critically. Studies relying on sleep diaries ought to be used with caution in this population and the addition of objective measurements such as actimetry appears to be advisable.

\section{Competing Interests}

Philipp S. Ritter, Cathrin Sauer, and Steffi Pfeiffer declare that they have no conflict of interests. Michael Bauer has received research support from the DFG (Deutsche Forschungsgemeinschaft) and BMBF (Bundesministerium für Bildung und Forschung). He is a consultant to AstraZeneca, Bristol-Myers Squibb, Merz, Janssen, Lilly, Servier, Takeda, Ferrer Int., Lundbeck, and Otsuka and has received speaking fees from AstraZeneca, Lilly, GlaxoSmithKline, Servier, Lundbeck, Otsuka, and Pfizer. Andrea Pfennig has received research support from GlaxoSmithKline and AstraZeneca. She has received speaking fees from AstraZeneca and Lilly.

\section{Authors' Contributions}

Philipp S. Ritter was responsible for conception, design, draft, execution of study, and interpretation of results. Cathrin Sauer was responsible for statistical analysis. Steffi Pfeiffer was responsible for database management. Michael Bauer was responsible for draft and critical revision. Andrea Pfennig was responsible for design of manuscript and draft.

\section{References}

[1] M. Bauer and A. Pfennig, "Epidemiology of bipolar disorders," Epilepsia, vol. 46, no. 4, pp. 8-13, 2005.

[2] A. G. Harvey, D. A. Schmidt, A. Scarnà, C. N. Semler, and G. M. Goodwin, "Sleep-related functioning in euthymic patients with bipolar disorder, patients with insomnia, and subjects without sleep problems," American Journal of Psychiatry, vol. 162, no. 1, pp. 50-57, 2005.

[3] P. S. Ritter, M. Höfler, H.-U. Wittchen et al., "Disturbed sleep as risk factor for the subsequent onset of bipolar disorderdata from a 10-year prospective-longitudinal study among adolescents and young adults," Journal of Psychiatric Research, vol. 68, pp. 76-82, 2015.

[4] G. Murray and A. Harvey, "Circadian rhythms and sleep in bipolar disorder," Bipolar Disorders, vol. 12, no. 5, pp. 459-472, 2010.

[5] T. H. Ng, K.-F. Chung, F. Y.-Y. Ho, W.-F. Yeung, K.-P. Yung, and T.-H. Lam, "Sleep-wake disturbance in interepisode bipolar disorder and high-risk individuals: a systematic review and meta-analysis," Sleep Medicine Reviews, vol. 20, pp. 46-58, 2015.

[6] E. Leibenluft, P. S. Albert, N. E. Rosenthal, and T. A. Wehr, "Relationship between sleep and mood in patients with rapidcycling bipolar disorder," Psychiatry Research, vol. 63, no. 2-3, pp. 161-168, 1996.

[7] T. Bschor, M. Ising, M. Bauer et al., "Time experience and time judgment in major depression, mania and healthy subjects. A controlled study of 93 subjects," Acta Psychiatrica Scandinavica, vol. 109, no. 3, pp. 222-229, 2004.

[8] R. Gonzalez, C. Tamminga, M. Tohen, and T. Suppes, "Comparison of objective and subjective assessments of sleep time in subjects with bipolar disorder," Journal of Affective Disorders, vol. 149, no. 1-3, pp. 363-366, 2013.

[9] K. A. Kaplan, L. S. Talbot, J. Gruber, and A. G. Harvey, "Evaluating sleep in bipolar disorder: comparison between actigraphy, polysomnography, and sleep diary," Bipolar Disorders, vol. 14, no. 8, pp. 870-879, 2012.

[10] J. Fernandez-Mendoza, S. L. Calhoun, E. O. Bixler et al., "Sleep misperception and chronic insomnia in the general population: role of objective sleep duration and psychological profiles," Psychosomatic Medicine, vol. 73, no. 1, pp. 88-97, 2011.

[11] P. S. Ritter, C. Marx, N. Lewtschenko et al., "The characteristics of sleep in patients with manifest bipolar disorder, subjects at high risk of developing the disease and healthy controls," Journal of Neural Transmission, vol. 119, no. 10, pp. 1173-1184, 2012.

[12] M. B. First, R. L. Spitzer, M. Gibbon, and J. B. Williams, Structured Clinical Interview for DSM-IV Axis I DisordersClinical Version, American Psychiatric Publishing, 1997.

[13] A. Schmidtke, P. Fleckenstein, W. Moises, and H. Beckmann, "Studies of the reliability and validity of the German version of the Montgomery-Asberg Depression Rating Scale (MADRS)," 
Schweizer Archiv für Neurologie und Psychiatrie, vol. 139, no. 2, pp. 51-65, 1988.

[14] S. A. Montgomery and M. Asberg, "A new depression scale designed to be sensitive to change," British Journal of Psychiatry, vol. 134, no. 4, pp. 382-389, 1979.

[15] R. C. Young, J. T. Biggs, V. E. Ziegler, and D. A. Meyer, "A rating scale for mania: reliability, validity and sensitivity," British Journal of Psychiatry, vol. 133, no. 11, pp. 429-435, 1978.

[16] M. Berk, F. Ng, W. V. Wang et al., "The empirical redefinition of the psychometric criteria for remission in bipolar disorder," Journal of Affective Disorders, vol. 106, no. 1-2, pp. 153-158, 2008.

[17] R. Dick, T. Penzel, I. Fietze, M. Partinen, H. Hein, and J. Schulz, "AASM standards of practice compliant validation of actigraphic sleep analysis from somnowatch ${ }^{\mathrm{TM}}$ versus polysomnographic sleep diagnostics shows high conformity also among subjects with sleep disordered breathing," Physiological Measurement, vol. 31, no. 12, pp. 1623-1633, 2010.

[18] C. Kühner, C. Bürger, F. Keller, and M. Hautzinger, "Reliabilität und validität des revidierten beck-depressionsinventars (BDIII). Befunde aus deutschsprachigen stichproben," Nervenarzt, vol. 78, no. 6, pp. 651-56, 2007.

[19] E. G. Altman, D. Hedeker, J. L. Peterson, and J. M. Davis, “The Altman self-rating mania scale," Biological Psychiatry, vol. 42, no. 10, pp. 948-955, 1997.

[20] C. Boudebesse, P. A. Geoffroy, F. Bellivier et al., "Correlations between objective and subjective sleep and circadian markers in remitted patients with bipolar disorder," Chronobiology International, vol. 31, no. 5, pp. 698-704, 2014.

[21] S. H. Jones, D. J. Hare, and K. Evershed, "Actigraphic assessment of circadian activity and sleep patterns in bipolar disorder," Bipolar Disorders, vol. 7, no. 2, pp. 176-186, 2005.

[22] L. L. Judd, H. S. Akiskal, P. J. Schettler et al., "The long-term natural history of the weekly symptomatic status of bipolar I disorder," Archives of General Psychiatry, vol. 59, no. 6, pp. 530537, 2002.

[23] W. K. Wohlgemuth, J. D. Edinger, A. I. Fins, and R. J. Sullivan Jr., "How many nights are enough? The short-term stability of sleep parameters in elderly insomniacs and normal sleepers," Psychophysiology, vol. 36, no. 2, pp. 233-244, 1999.

[24] A. Sadeh, "The role and validity of actigraphy in sleep medicine: an update," Sleep Medicine Reviews, vol. 15, no. 4, pp. 259-267, 2011. 


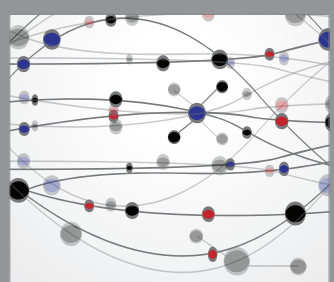

The Scientific World Journal
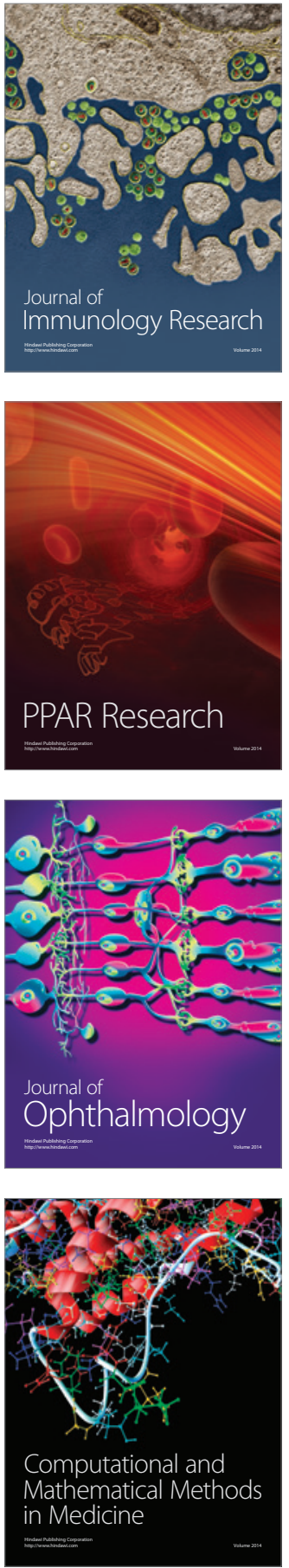

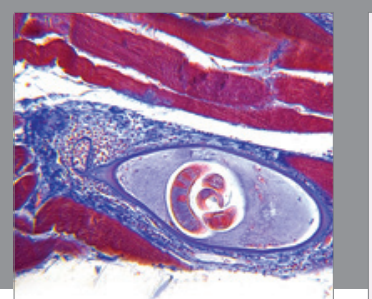

Gastroenterology Research and Practice

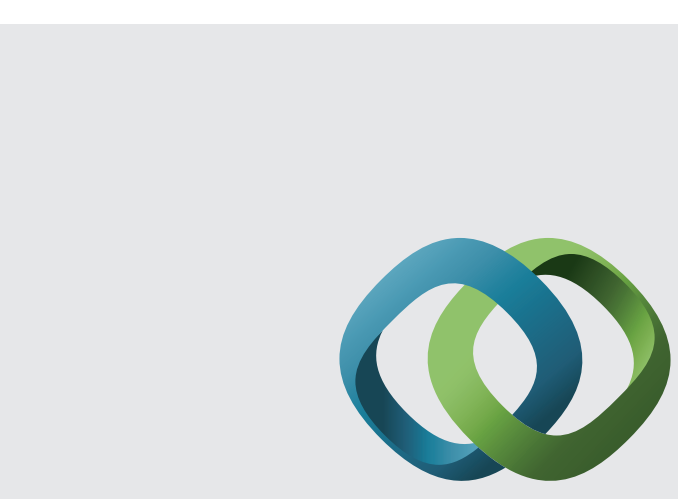

\section{Hindawi}

Submit your manuscripts at

http://www.hindawi.com
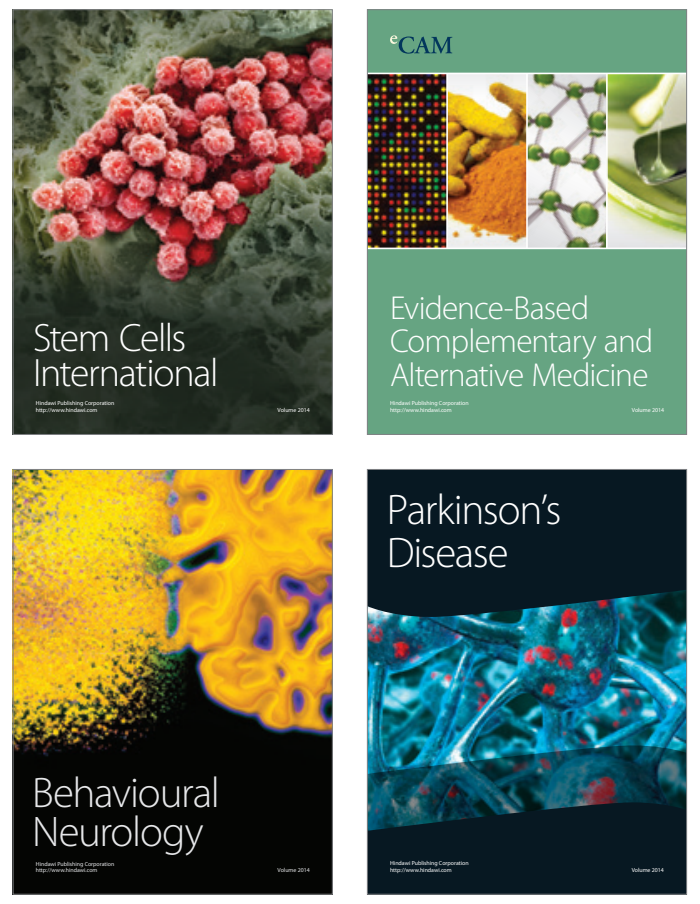
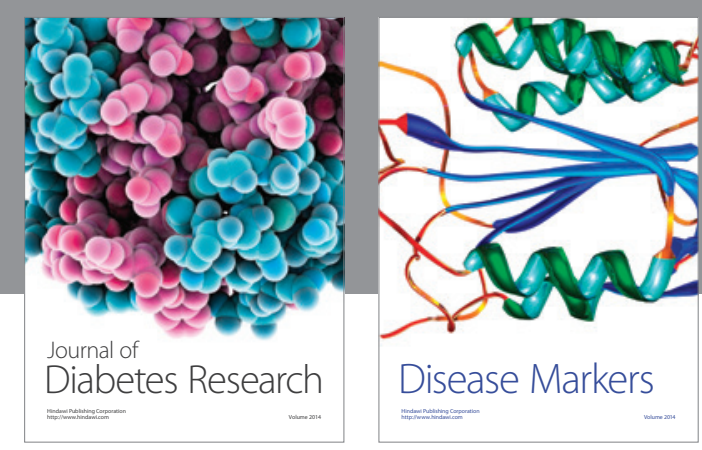

Disease Markers
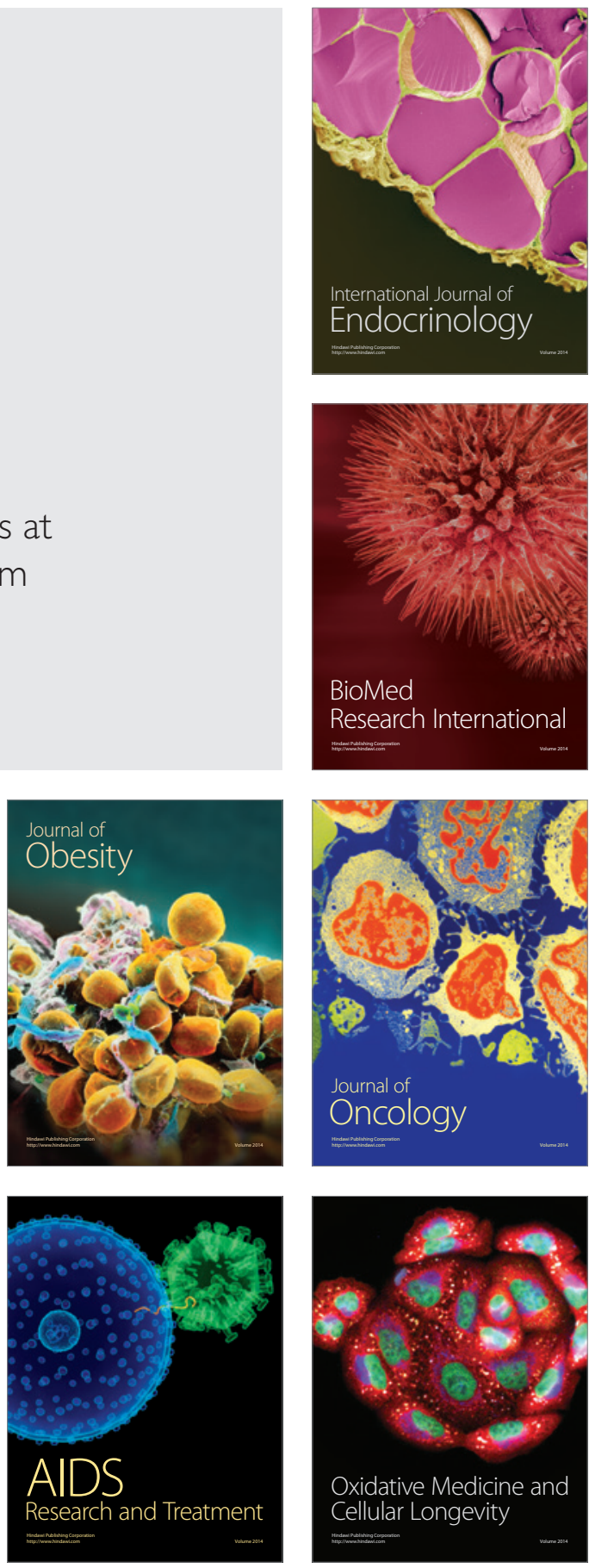